\title{
Diagnostic yield and management benefit of laparoscopy: a prospective audit
}

\author{
G C Vander Velpen, S M Shimi, A Cuschieri
}

\begin{abstract}
A prospective audit of the diagnostic yield and management benefit of laparoscopy was undertaken in 220 consecutive patients. The procedure was performed electively in 180 patients and as an emergency in 40. The indications for laparoscopy in the elective group were suspected hepatic disease, staging of intra-abdominal malignancy, diagnostic problems, and chronic abdominal pain. Emergency laparoscopy was performed in patients admitted with acute abdominal pain. Diagnostic benefit varied with the indication for the procedure: liver disease $71 \%$, tumour staging $87 \%$, uncertain diagnosis $74 \%$, acute abdominal pain $100 \%$, and chronic abdominal pain $41 \%$. Clinical management was significantly influenced by laparoscopy in 15 of $21(71 \%)$ patients with liver disease, 10 of $30(33 \%)$ with intra-abdominal malignancy, 5 of 19 $(26 \%)$ with uncertain diagnosis, 32 of 40 $(80 \%)$ with acute abdominal pain, and 15 of $110(23 \%)$ patients with chronic abdominal pain. A wrong assessment of the nature or stage of the disease was made by laparoscopy in 3 of $220(1 \cdot 0 \%)$. There was no morbidity or mortality attributed to laparoscopy in the study.
\end{abstract}

(Gut 1994; 35: 1617-1621)

Although laparoscopy was introduced at the beginning of this century, ${ }^{1}$ it has, until recently, failed to gain widespread acceptance largely because of the availability of non-invasive imaging techniques. ${ }^{23} \mathrm{~A}$ definitive diagnosis is not always possible, however, with non-invasive imaging tests including contrast radiology, computed tomography, magnetic resonance imaging, and ultrasonography. ${ }^{45}$

New technical advances such as contact ultrasound scanning with linear array high resolution laparoscopic probes 6 have enhanced the diagnostic yield of laparoscopy. In addition, target needle biopsy and fine needle cytology carried out under laparoscopic guidance permit definitive diagnosis of suspect lesions. ${ }^{78}$ In patients with intra-abdominal malignancy, the benefit from laparoscopy often extends beyond establishing a diagnosis as the procedure provides invaluable information on staging and often identifies inoperability, thereby avoiding needless laparotomy. ${ }^{9-14}$ In this respect, laparoscopy is the only reliable method for the detection of peritoneal deposits. ${ }^{9-12} 1516$ In many of these incurable patients, palliative surgery when indicated, can also be undertaken by the laparoscopic approach. ${ }^{17}$

The aim of this prospective study was to evaluate the diagnostic yield by clinical indication and to determine the management benefit accruing from diagnostic laparoscopy in the various groups.

\section{Patients and methods}

The prospective audit was undertaken in a surgical professional unit with a special interest in gastroenterology and hepatobiliary disease. The protocol was formulated on an existing unit policy for laparoscopy established for many years. In particular, laparoscopy was only undertaken when clinical assessment and routine imaging tests failed to provide all the necessary information needed for individual patient management. In all instances, laparoscopy was carried out in the operating theatre under general anaesthesia. Ancillary diagnostic measures such as laparoscopic target needle or punch biopsy, cytology or cholangiography were performed whenever dictated by the laparoscopic findings. In patients with suspected pancreatic disease, visualisation of the pancreas was conducted by the infragastric approach. ${ }^{10} 18$ Contact laparoscopic ultrasound scanning of the liver and pancreas with high resolution $7 \cdot 5 \mathrm{Mhz}$ linear array probes (Aloka, Keymed, UK) became available to the team only during the last few months of the study, which was conducted between January 1990 and December 1992. During this period 220 consecutive patients: 62 males, 158 females, mean age 42 years, range $10-81$, had assessment by laparoscopy (Table I).

For the purpose of the study, a positive diagnostic benefit was defined as a definite diagnosis made on the basis of the laparoscopic findings or the reliable exclusion of organic disease by the procedure. In the case of tumour staging diagnostic benefit also implied assessment of inoperability either because of metastatic deposits or significant local invasion.

The patient was deemed to have benefited from laparoscopy whenever the findings of this investigation influenced the clinical decision

TABLE I Patient groups

\begin{tabular}{lc}
\hline Patient groups & No of patients \\
\hline Suspected liver disease & 21 \\
Tumour staging & 30 \\
Uncertain diagnosis & 19 \\
Acute abdominal pain & 40 \\
Chronic abdominal pain & 110 \\
Total & 220 \\
\hline
\end{tabular}

Department of
Surgery, University of Dundee, Ninewells School, Dundee G C Vander Velpen 9 February 1994
} 
TABLE II Histopathological diagnosis of liver biopsy specimens in patients with suspected liver disease

\begin{tabular}{ll}
\hline Histopathology & No of patients \\
\hline Cirrhosis & 7 \\
Fatty liver & 1 \\
Coxsackie B hepatitis & 1 \\
Solitary hepatic cyst & 2 \\
Metastatic adenocarcinoma & 1 \\
Cholangiocarcinoma & 1 \\
Hepatocellular carcinoma & 1 \\
Normal & 6 \\
\hline
\end{tabular}

on the treatment in the individual case. The validity of the laparoscopic evaluation was assessed by follow up, which was for a minimum of six months in every patient.

\section{Results}

There was no mortality or morbidity associated with the procedure. The results are described separately for each group of patients according to the indication for diagnostic laparoscopy.

GROUP 1: SUSPECTED LIVER DISEASE

All patients with suspected liver disease were subjected to laparoscopy. This policy reflects a long established practice in the unit and is based on three advantages of the laparoscopic approach: macroscopic assessment of the liver, increased safety of liver biopsy for diffuse disease (including multiple biopsies), and the ability for precise target biopsy of focal lesions. Twenty one patients included in the study were in this category: abnormal liver chemistry $n=13$, focal liver lesion identified by ultrasound $\mathrm{n}=6$, toxicity after drug treatment (methotrexate) $n=1$, and portal hypertension of unknown aetiology $n=1$.

Laparoscopic guided biopsy specimens were obtained from 20 of 21 patients. One patient was found to have a hepatic haemangioma and no specimen was taken. Table II shows the histological findings. A definitive diagnosis was established in 14 of 20 cases $(70 \%)$. In six cases the result of diagnostic laparoscopy did not positively influence patient management. Included in this group are five patients with abnormal liver chemistry and one patient with portal hypertension of unknown aetiology. In all these, the liver biopsy specimen was either normal or showed mild, non-specific changes; and laparoscopic inspection failed to identify any definitive abnormality. Table III shows the comparison between laparoscopic findings and histological diagnosis. In two patients, histological examination of biopsy material did not confirm the laparoscopically detected cirrhosis.

Laparoscopic transhepatic cholangiography was performed in one patient with obstructive

TABLE III Comparison between laparoscopic and histological diagnoses in patients with liver disease

\begin{tabular}{|c|c|c|c|}
\hline & Positive histology & Negative histology & Total \\
\hline $\begin{array}{l}\text { Positive } \\
\text { laparoscopy } \\
\text { Negative } \\
\text { laparoscopy }\end{array}$ & $\begin{array}{l}13 \\
\text { true positive } \\
1 \\
\text { false negative }\end{array}$ & $\begin{array}{l}2 \\
\text { false positive } \\
4 \\
\text { true negative }\end{array}$ & $\begin{array}{r}15 \\
5\end{array}$ \\
\hline
\end{tabular}

jaundice and two patients had laparoscopic deroofing of solitary hepatic cysts.

GROUP 2: TUMOUR DIAGNOSIS AND STAGING

Patients were subjected to laparoscopy only when the routine imaging tests including computed tomography failed to provide the necessary information either for the diagnosis of intra-abdominal malignant disease (primary or metastatic disease) or the resectability of the primary (staging) when this originated in an intraperitoneal organ. There were 30 patients in this category: gastric cancer $n=10$, oesophageal cancer $n=5$, breast cancer $n=4$, pancreatic cancer $n=8$, ovarian cancer $n=1$, cerebral tumour $n=1$, and lymphoma $n=1$. This cohort accounted for $19 \%$ of patients with intra-abdominal cancer admitted to the unit during the period of the study.

Routine non-invasive assessment (radiology, computed tomography, and ultrasound) suggested inoperability in only one of 10 patients with gastric cancer. Seven of these patients were judged to have resectable disease by laparoscopy. Six subsequently had a potentially curative resection but in one patient the cancer was found to be unresectable at laparotomy because of extensive posterior local infiltration undetected by laparoscopy. Advanced disease was shown by laparoscopy in three patients: local infiltration $n=3$, peritoneal or hepatic metastases $n=3$. Metastatic gastric adenocarcinoma was confirmed by target needle or punch biopsies in two of these patients. Laparotomy was avoided in all three.

Four of five patients with cancer of the lower oesophagus had no macroscopic evidence of intra-abdominal disease at laparoscopic staging and normal lavage cytology (class I mesothelial cells). All four patients subsequently had an oesophagectomy. One patient who was found to have multiple hepatic metastases at laparoscopy confirmed by target liver biopsy was treated by endoscopic intubation of the tumour.

The lymphoma was diagnosed by computed tomography as a large left retroperitoneal mass. Laparoscopy confirmed the presence of a large tumour in the left paracolic space between the spleen and the left kidney. In addition, the spleen and liver were found to contain tumour deposits. Sufficient material was obtained from the primary lesion to permit histological cell typing and grading (high grade B type malignant lymphoma).

Eight patients had a laparoscopy for staging of pancreatic or periampullary cancer. Five had evidence of metastatic disease on the noninvasive imaging tests, which was confirmed by laparoscopy (hepatic deposits). In three of these patients, additional peritoneal seedling undetected by computed tomography or ultrasound imaging was confirmed by laparoscopy. Metastatic hepatic and peritoneal disease was only discovered by laparoscopy in another patient. This and three other patients in this series had palliative treatment for the jaundice by laparoscopic bilioenteric bypass.

For six patients, undergoing laparoscopy for 
suspected recurrence of previously treated malignancy, laparoscopy confirmed hepatic metastatic disease in three, benign disease (hepatic cyst in one), and was normal in two. In view of the persistent abnormality on repeat scanning, one of these patients was subsequently subjected to a laparotomy when a large solitary metastatic deposit was found posteriorly in segment VIII of the liver.

GROUP 3: DIAGNOSTIC PROBLEMS

In nineteen patients diagnostic laparoscopy was carried out in an attempt to establish a diagnosis, which remained uncertain after the routine imaging tests: undiagnosed intraabdominal mass $n=6$, suspected pancreatic lesion $n=6$, suspected intra-abdominal malignancy $\mathrm{n}=3$, recurrent occult gastrointestinal bleeding $n=2$, and unconfirmed inflammatory bowel disease $n=2$. These patients did not have percutaneous ultrasound guided needle biopsy as we prefer the visually targeted tissue sampling at laparoscopy.

Diagnostic laparoscopy could determine the nature of the computed tomography ultrasound detected masses in five of six patients: large mesenteric cyst (deroofed laparoscopically), ovarian cancer with widespread intraabdominal disease $\mathrm{n}=2$, primary splenic lymphoma (B cell), and one patient with a postappendicectomy chronic inflammatory mass at the caecal pole with no evidence of inflammatory bowel disease. No abnormality was detected by laparoscopy in the sixth patient in whom the ultrasound had suggested a mass in the right iliac fossa.

Laparoscopy with examination of the pancreas using the infracolic technique was undertaken in six patients suspected of pancreatic lesion as a result of computed tomography or ultrasound scanning. In all patients, the pancreas was visualised and palpated from head to tail. Laparoscopic ultrasound contact scanning of the pancreas was performed in the last two patients. Pancreatic abnormality was confirmed by laparoscopy in three patients. Two had chronic pancreatitis, one with a pseudocyst. In one patient, an insulinoma behind the body of the pancreas at the junction of splenic with the portal vein was located by laparoscopic contact ultrasound scanning. The insulinoma though biochemically confirmed, could not be located

TABLE IV Postlaparoscopy diagnosis in patients with acute abdominal pain

\begin{tabular}{ll}
\hline Diagnosis & No of patients \\
\hline Acute appendicitis & 13 \\
Complications of ovarian cysts & 9 \\
Pelvic inflammatory disease & 2 \\
Ectopic pregnancy & 1 \\
Retrograde menstruation & 1 \\
Endometriosis & 2 \\
Mesenteric adenitis & 1 \\
Non-specific right iliac fossa pain & 6 \\
Curtis-Fitz-Hugh syndrome & 1 \\
Acute cholecystitis & 1 \\
Acute pancreatitis & 1 \\
Gastroenteritis & 1 \\
Adhesive small bowel obstruction & 1 \\
\hline
\end{tabular}

by repeated angio computed tomography before the laparoscopy. In three patients, the pancreas was normal.

Ovarian cancer was found in one of three patients with suspected intra-abdominal malignancy based on unexplained weight loss and malaise. No laparoscopic abnormality was found in two patients with multiple negative investigations in whom the diagnosis has remained uncertain despite follow up.

Two patients had been extensively investigated for recurrent occult gastrointestinal bleeding with contrast radiology, endoscopy, angiography, and radiolabelled red cell scans with negative results. Two angiomas of the jejunum were discovered by laparoscopy in one patient. These were suture ligated laparoscopically with cessation of bleeding episodes. In the other patient no other abnormality other than diverticulitis was found. This patient underwent subsequent sigmoid colectomy and has had no further episodes of rectal bleeding. Two patients with Crohn's disease (distal ileum) were confirmed laparoscopically after contrast investigations proved inconclusive. In one of these, the lesion was adherent to the sigmoid colon.

\section{GROUP 4: ACUTE ABDOMINAL PAIN}

Laparoscopy was performed in 40 patients, 32 females, admitted as an emergency with an acute abdomen disorder in whom clinical assessment and routine emergency tests including scout abdominal films or emergency ultrasound examination failed to provide a firm diagnosis, or when doubt remained as to the need for surgical intervention. The pain was situated in the right iliac fossa in 35 , in the right upper quadrant in three, and was generalised in two. Table IV shows the various diagnoses established by laparoscopy in this group.

In terms of patient management, emergency laparoscopy permitted triage of these patients into three groups: those requiring urgent surgical intervention $(n=15)$, those with acute disease requiring conservative treatment $(n=17)$, and those without an acute abnormality $(n=8)$. The laparoscopic assessment was correct in all the cases $(n=15)$ and when surgical removal of an inflamed organ (appendix, gall bladder) was undertaken, histological examination of the excised specimen confirmed the diagnosis in every case. Likewise, the decision on conservative treatment or delayed elective intervention based on the laparoscopic assessment did not require revision in any of the patients $(n=25)$.

\section{GROUP 5: CHRONIC ABDOMINAL PAIN}

This was the largest group $(n=110)$ and contained a predominance of women $(n=86)$. The mean age was 39 years, with a range 14-74. The indication was either chronic abdominal pain with or without associated bowel upset, or dyspeptic symptoms. All patients had extensive investigations that failed to determine the cause of their complaint. Ninety six of 110 patients were referred by 
TABLE V Postlaparoscopy diagnosis in patients with chronic abdominal pain

\begin{tabular}{ll}
\hline Diagnosis & No of patients \\
\hline Adhesions & 22 \\
Diverticulosis itis & 4 \\
Colitis & 4 \\
Salpingitis & 4 \\
Sabuacute appendicitis & 2 \\
Cholecystolithiasis & 1 \\
Endometriosis & 1 \\
Ovarian cancer & 1 \\
Focal nodular hyperplasia & 1 \\
Pancreatic cancer & 1 \\
Curtis-Fitz-Hugh syndrome & 1 \\
Distal ileitis & 1 \\
Chronic duodenal ulceration & \\
\hline
\end{tabular}

gastroenterologists and 14 patients by other medical specialities.

Positive findings were recorded in 45 patients $(41 \%)$. This included 23 patients with intra-abdominal adhesions and 22 patients with a significant specific abnormality, which was treated with subsequent relief of their symptoms (Table V). No abnormality was found on laparoscopic inspection of the peritoneal cavity and contents in 65 patients $(59 \%)$.

In the group with positive findings $(n=45)$, 25 patients $(56 \%)$ received laparoscopic treatment: adhesiolysis $\mathrm{n}=21$, cholecystectomy $\mathrm{n}=2$, and appendicectomy $\mathrm{n}=2$. Five of the 45 patients $(11 \%)$ required a laparotomy: adhesiolysis and mesenteric plication $\mathrm{n}=2$, right hemicolectomy $n=1$, left hepatic lobectomy $\mathbf{n}=1$, and bilateral oophorectomy and omentectomy $n=1$. In the study laparoscopic adhesiolysis was undertaken to ensure complete inspection of the peritoneal cavity and its contents and not to evaluate the efficacy of pain relief by laparoscopic adhesiolysis. In any event, most patients $(n=18)$ continued to experience abdominal pain and only three patients with episodes of colic and abdominal distension obtained relief from the procedure in the short term.

\section{OVERALL DIAGNOSTIC YIELD AND}

MANAGEMENT BENEFIT

Table VI summarises the diagnostic yield and influence on patient management obtained by laparoscopy.

\section{Discussion}

The results of this prospective audit have confirmed the reported retrospective experience on the diagnostic yield of laparoscopy in surgical gastroenterological practice. ${ }^{2-5} 710-1619-23$ Furthermore, it has verified the management benefit of this procedure. Both the diagnostic yield and the influence on clinical management vary with the indication for the laparoscopy. The incidence of a missed abnormality was low and will probably be reduced further by the routine use of laparoscopic contact ultrasound examination. Laparoscopic ultrasonography permits the detection of intraparenchymal lesions in the solid organs not detected by visual inspection and in the case of pancreatic tumours, permits detection of vascular encasement indicative of local inoperability. ${ }^{24} 25$

Laparoscopy permits visual inspection of both focal and diffuse disease of the liver and the detection of portal hypertension and ascites. Target biopsy is performed under visual guidance with increased safety and higher diagnostic yield when compared with the equivalent percutaneous procedures. ${ }^{7-9}$ The diagnostic accuracy of percutaneous liver biopsy is limited by a significant random variability even in the presence of diffuse disease. ${ }^{26}$ In a randomised prospective trial comparing blind with laparoscopic target biopsy, cirrhosis was missed in $20 \%$ of cases by blind percutaneous technique. ${ }^{9}$ In both focal and diffuse neoplastic disease the diagnostic yield of target biopsy under visual guidance is double that of the blind technique. ${ }^{8}$ In this study diagnosis of an abnormality was made in $100 \%$ of patients with focal hepatic lesions initially detected by computed tomography or ultrasound.

Although the reported detection of focal hepatic lesions by computed tomography is high, this imaging test carries a $10 \%$ false positive and negative rate ${ }^{27}$ and confirmation in the individual patient requires visual inspection and biopsy for histological confirmation. ${ }^{19} 20$ Currently there is no imaging or laboratory test that can evaluate with sufficient accuracy and reliability the resectability of intra-abdominal tumours. ${ }^{28}$ Several studies have reported the usefulness and reliability of laparoscopy in the detection of advanced inoperable disease. ${ }^{10-16}$ Furthermore, in patients suffering from pancreatic cancer, surgery to relieve the obstructive jaundice and, when present duodenal obstruction, can be conducted laparoscopically, thereby avoiding the complications associated with stent insertion. ${ }^{17}$

By virtue of the similar density of the pancreatic parenchyma to surrounding fat, lesions of this organ are notoriously difficult to detect by computed tomography. Wholly intrapancreatic lesions are thus often missed unless dilatation of the biliary tree is present. ${ }^{29} 30$ Repeat scanning after a suitable interval is often performed for suspect lesions but this practice causes undue anxiety and often leads to a diagnostic laparotomy when disease exclusion remains uncertain. The entire pancreas can be fully inspected laparoscopically by the infracolic technique ${ }^{101118}$ and its parenchyma scanned with high resolution linear array ultrasound probes. ${ }^{25}$ Lesions identified by inspection or ultrasound scanning are further examined by fine needle aspiration cytology. Although all the instances of pancreatic and

TABLE VI Diagnostic and management benefit of laparoscopy

\begin{tabular}{llll}
\hline & \multicolumn{2}{l}{ Diagnostic } & management \\
\cline { 2 - 3 } Patient group & Yield & Benefit & Disadvantage \\
\hline Liver disease & $15 / 21$ & $15 / 21$ & $1 / 21$ \\
Tumour staging & $26 / 30$ & $10 / 30$ & $2 / 30$ \\
Uncertain diagnosis & $14 / 19$ & $5 / 19$ & 0 \\
Acute abdominal pain & $40 / 40$ & $32 / 40$ & 0 \\
Chronic abdominal pain & $45 / 110$ & $25 / 110$ & 0 \\
\hline
\end{tabular}


periampullary cancers were diagnosed by endoscopic retrograde cholangiopancreatography in this study, this procedure did not provide adequate information on staging and resectability of the disease.

In patients with acute abdominal pain and uncertain diagnosis, laparoscopy permits identification of those patients who require emergency or urgent surgery and those who should be treated conservatively. In this last group hospital stay needed for observation is reduced. Laparoscopy is particularly useful in women of child bearing age in whom tuboovarian abnormality often presents with acute right lower abdominal pain simulating acute appendicitis. Some $15 \%$ of patients with acute abdominal pain who are subjected to an unnecessary appendicectomy suffer complications of the operation which, in women, may result in infertility. ${ }^{31}$ For this reason, it has been our policy to avoid removal of macroscopically normal looking appendixes and we have not encountered a single instance when this policy resulted in missed acute disease. By contrast, all laparoscopically diagnosed cases were confirmed as acute appendicitis by histological examination of the excised specimen.

In common with other reports 2223 the lowest diagnostic yield was encountered in patients with chronic abdominal pain. Even so, significant abnormality was found in some of these patients including two patients with undiagnosed cancer. The data obtained in this group support a policy of early laparoscopy in preference to multiple and expensive investigations before recourse to this examination. Laparoscopic adhesiolysis was performed in this study to permit full inspection of the peritoneal cavity and its contents. It was not the intention of the study to evaluate the efficacy of laparoscopic division of adhesions in the relief of chronic abdominal pain, although our impression has been that pain is unaffected except when it is colicky in nature and accompanied by abdominal distension.

The main message of this study is that there are no specific indications for laparoscopy in gastroenterology and hepatobiliary disease as such. As with flexible endoscopy, it should be used regularly but selectively in problematic cases. Certainly, the occasional recourse to diagnostic laparoscopy is unlikely to be useful or safe. Soon, hopefully, gastroenterologists and hepatologists will come to look at diagnostic laparoscopy in the same light as flexible endoscopy and thereby improve their diagnostic accuracy and patient management and in addition, avoid a battery of expensive imaging tests in problematic cases.
1 Keeling G. Bur coelioskopie. Arch Klin Chir 1923; 126: 226-9.

2 Gandolfi L, Rossi A, Leo P, Solmi L, Muratori R. Indications for laparoscopy before and after the introduction of ultrasonography. Gastrointest Endosc 1985; 31: 1-3.

3 Dagnini G. Laparoscopy and imaging techniques. SpringerVerlag: Berlin, Heidelberg 1990.

4 Morl M. Laparoscopy. Present situation and prospects. Endoscopy 1987; 19: 167-9.

5 Lightdale CJ. Laparoscopy in the age of imaging. Gastrointest Endosc 1985; 1: 47-8.

6 Fornari F, Civardi G, Cavanna L, et al. Laparoscopic ultrasonography in the study of liver disease. Preliminary sonography in the study of liver

7 Irving AD, Cuschieri A. Laparoscopic assessment of the jaundiced patient: a review of 53 patients. Br $\mathcal{F}$ Surg 1978; 65: 678-80

8 Jori PJ, Peschle C. Combined peritoneoscopy and liver biopsy in the diagnosis of hepatic neoplasm. Gastroenterol 1972; 63: 1016-9.

9 Pagliaro al, Arinaldi F, Craxi A, et al. Percutaneous blind biopsy versus laparoscopy with guided biopsy in diagnosis in cirrhosis. Dig Dis Sci 1983; 28: 39-43.

10 Cuschieri A, Hall AW, Clark J. Value of laparoscopy in the diagnosis and management of pancreatic cancer. Gut 1978; 19: 672-7.

11 Cushieri A. Laparoscopy for pancreatic cancer: does it benefit the patient? Eur $\mathcal{F}$ Surg Oncol 1988; 14: 41-4

12 Warshaw AL, Tepper JE, Shipley WU. Laparoscopy in the staging and planning of therapy for pancreatic cancer. Am ₹ Surg 1986; 151: 76-9.

13 Possik RA, Franco EL, Pires DR, et al. Sensitivity, specificity and predictive value of laparoscopy for the staging of gastric cancer and for the detection of liver metastases. Cancer 1986; 58: 1-6.

14 Shandall A, Johnson C. Laparoscopy or scanning in oesophageal and gastric carcinoma? Br f Surg 1985; 72: 449-51.

15 Ishida H, Furukawa Y, Kuroda H, et al. Laparoscopic observation and biopsy of the pancreas. Endoscopy 1981; 13: $68-73$.

16 Ishida H. Peritoneoscopy and pancreas biopsy in the diagnosis of pancreatic disease. Gastrointest Endosc 1983; 29: $211-8.16$

17 Shimi SM, Banting SB, Cuschieri A. Laparoscopy in the management of pancreatic cancer: endoscopic cholecystojejunostomy for advanced disease. Br $\mathcal{F}$ Surg 1992; 79: 317-9.

18 Strauch M, Lux G, Ottenjann R. Infragastric pancreascopy. Endoscopy 1973; 5: 30-2.

19 Herrera JL, Brewer TG, Peura DA. Diagnostic laparoscopy: A prospective review of 100 cases. Am $\mathcal{F}$ Gastroenterol 1989; 84: $1051-4$.

20 Lightdale CJ. Clinical applications of laparoscopy in patients with malignant neoplasms. Gastrointest Endosc 1982; 28: 99-101.

21 DeVita VT, Bagley CM, Goodell B, O'Kieffe DA, Trujillo NP. Peritoneoscopy in the staging of Hodgkin's disease. Cancer Research 1971; 31: 1746-50.

22 Lewis A, Archer TJ. Laparoscopy in general surgery. Br f Surg 1981; 68: 778-80.

23 Nagy AG, James D. Diagnostic laparoscopy. Am f Surg $1989 ; 157: 490-3$.

24 Miles WFA, Paterson-Brown, Garden OJ. Laparoscopic contact hepatic ultrasonography. $B r \mathcal{F}$ Surg 1992; 79: 419-20.

25 Pietrabissa A, Shimi S, Cushieri A. Localization of insulinoma by laparoscopic infragastric inspection of the insulinoma by laparoscopic infragastric inspection of the pancreas

26 Abdi W, Millan J, Mezey E. Sampling variability on percutaneous liver biopsy. Arch Int Med 1979; 139: 667-8.

27 Scherer U, Rothe R, Eisenburg J, et al. Diagnostic accuracy of CT in circumscript liver disease. Am $\mathcal{F}$ Roentgenol 1978; 130: $711-4$

28 Kemeny MM, Sugarbaker P, Smith TJ, et al. A prospective analysis of laboratory tests and imaging studies to detect hepatic lesions. Ann Surg 1982; 195: 163-7.

29 Haaga JR, Alfidi RJ, Havrilla TR, et al. Definitive role of CT scanning of the pancreas. The second year's experience. scanning of the pancreas. The

30 Sheedy PF, Stephens DH, Hattery RR, MacCarty RL. Computed tomography in the evaluation of patients with Computed tomography in the evaluation of patients with suspected carci $731-7$.

31 Lewis FR, Holcroft JW, Boey J, Dunphy JE. Appendicitis, a critical review of diagnosis and treatment in 1000 cases. Arch Surg 1975; 110: 677-84. 